\title{
Mapping Inequality in SARS-CoV-2 Household Exposure and Transmission Risk in the USA
}

J Gen Intern Med 36(5):1476-8

DOI: $10.1007 / \mathrm{s} 11606-021-06603-0$

(C) Society of General Internal Medicine 2021

\section{INTRODUCTION}

As COVID-19 vaccines become available, public health departments will have numerous options for pandemic control. While supply constraints will limit early vaccine availability, another concern is that vaccine uptake may be undermined by barriers to access and vaccine hesitancy. Communities of color may be most susceptible to low coverage due to long-standing disparities in healthcare, mistrust fueled by a history of exploitation in clinical trials, and other structural risk factors. ${ }^{1}$ At the same time, accumulated evidence on SARS-CoV-2 transmission suggests that low-income, essential service workers face higher rates of community exposure and may be unable to self-isolate under threat of income or job loss. ${ }^{2}$ Crowded housing can extend infection risks to other household members. ${ }^{2}$ The legacy of residential segregation contributes to geographic concentration of these risks. ${ }^{3}$ Given this combination of factors, prioritizing outreach and prevention efforts toward communities with identifiable exposure risks may enable greater impact in mitigating harms and reducing disparities. To inform such prioritization, we characterize and map populations facing higher risk of exposure and transmission due to occupation and housing characteristics.

\section{METHODS}

We used the American Community Survey five-year (20142018) Public Use Microdata Sample to estimate the number and percentage of people living in households with higher risk of SARS-CoV-2 expoosure and transmission ("high-risk"). ${ }^{4}$ We defined high-risk households as those with (1) fewer rooms than people and (2) at least one essential worker. We identified essential workers using Standard Occupational Classification codes. ${ }^{5}$ Using provided weights, we produced representative estimates for 2351 Public Use Microdata Areas

Received November 25, 2020

Accepted January 1, 2021

Published online February 18, 2021
(PUMAs), the smallest geographic unit of analysis available. For counties with a population over 100,000, PUMAs allow analysis at the sub-county level.

Analyses were conducted using R version 4.0.2. Data and code are available at https://github.com/SC-COSMO/household_exposure_risk.

\section{RESULTS}

17.7 million people (5.6\% of the US population) live in households with at least one essential worker and fewer rooms than people. Fifty-eight percent of people living in these households have total family income below $200 \%$ of the Federal Poverty Level and $31.2 \%$ live in multigenerational households ( $\geq 3$ generations). Across 2351 PUMAs, the percent of people living in high-risk households ranges from 0.3 to $43.2 \%$ (median: $3.8 \%$ ). Areas with high proportions of people living in high-risk households are dispersed across the country; 37 states have at least one PUMA in the highest quartile of risk $(>6.6 \%)$. There is excess risk concentration even within relatively small geographic areas, and therefore county-level prioritization can hide substantial inequality and heterogeneity in risk, particularly in large metropolitan areas (Fig. 1, inset).

People of color (POC; 39\% of the total population) account for $76 \%$ of those in high-risk households. Eight hundred twenty-five PUMAs (35\%) across 48 states have $>10 \%$ of their POC population living in highrisk households, compared to 26 PUMAs (1\%) in six states for non-Hispanic White populations (Fig. 1). In $96 \%$ of PUMAs, a greater proportion of the POC population live in high-risk households compared to the non-Hispanic White population (mean ratio: 5.5).

\section{DISCUSSION}

Our study maps one dimension of structural inequality, which is closely linked to transmission risk and health disparities, and can be useful to health departments seeking to prioritize prevention efforts. Areas with high proportions of people living in high-risk households are disproportionately communities of color. Overcoming long-standing barriers to uptake of vaccines and other preventive services will likely require focused efforts in 

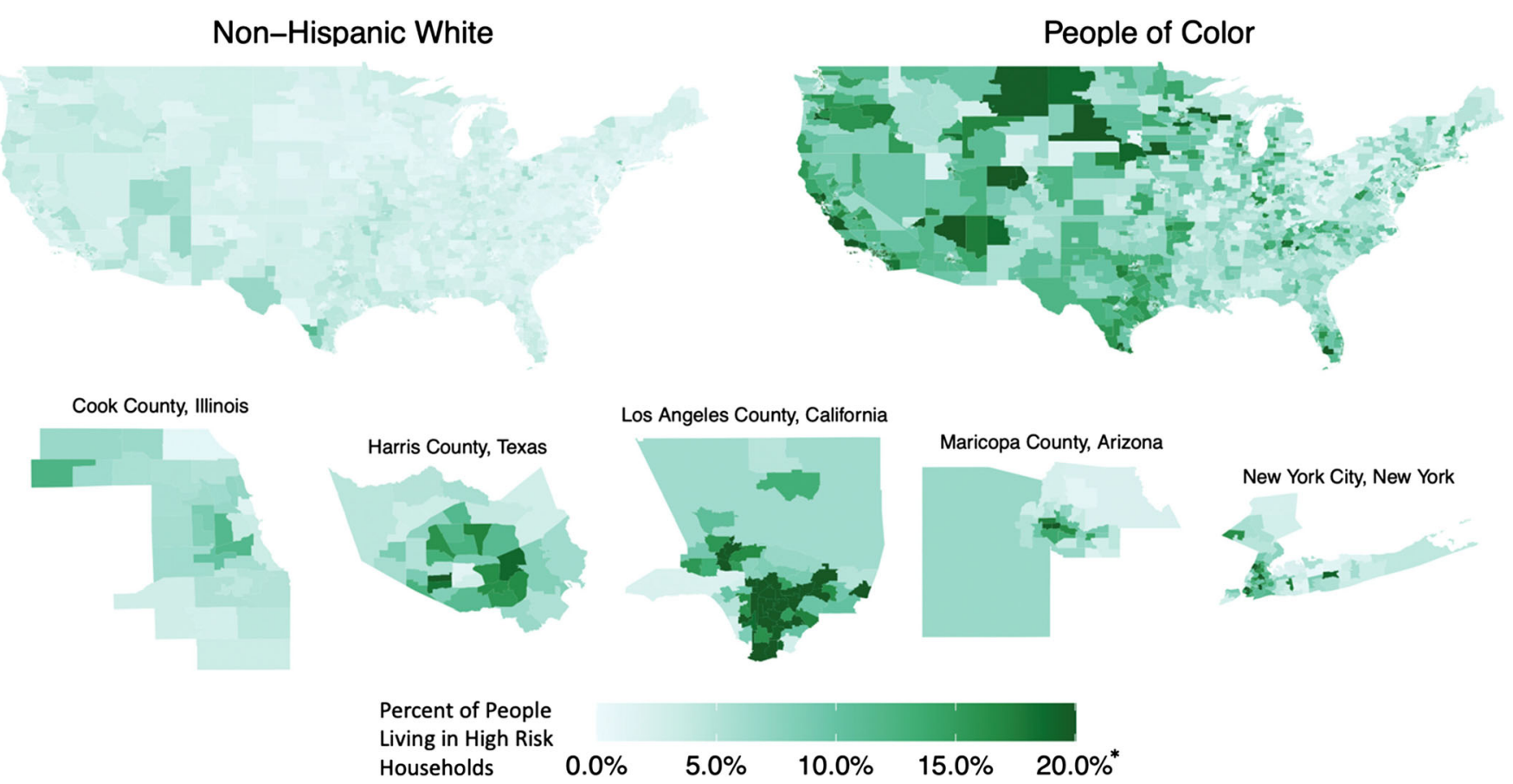

Figure 1 Percent of people living with at least one essential worker in a household with fewer rooms than people, by PUMA and race/ethnicity. Inset shows estimates for the four most-populated counties and New York City. Colors are top-coded at 20\%. An asterisk indicates that 132 PUMAs were above $20 \%$ and were top-coded in the People of Color map. Three PUMAs were top-coded in the non-Hispanic White map. Thirty-nine PUMAs were top-coded in the county inset map.

these areas led by local health departments and community organizations. ${ }^{6}$

Most individuals living in households with an essential worker and more people than rooms have low income, and many live in multigenerational households, where older members have higher risk of severe COVID-19 outcomes. Policies are needed to address barriers to self-isolation, for example paid sick-leave and job protection for those needing to isolate, cash assistance during isolation, and temporary housing for those unable to safely isolate at home. Scaled-up communitybased testing to address healthcare access barriers will be another important dimension of an effective response. By preferentially focusing prevention efforts, including testing, supported isolation, and active promotion of vaccine uptake in areas where high-risk households are concentrated, an equity-informed COVID-19 response can help to mitigate health disparities in communities of color and control outbreaks nationally.

Marissa B. Reitsma, BS ${ }^{1}$

Joshua A. Salomon, $P h D^{1}$

Jeremy D. Goldhaber-Fiebert, $P h D^{1}$

${ }^{1}$ Stanford Health Policy, Centers for Health Policy and Primary Care and Outcomes Research, Department of Medicine, Stanford University, 615 Crothers Way, Stanford, CA 94305, USA
Corresponding Author: Marissa B. Reitsma, BS; Stanford Health Policy, Centers for Health Policy and Primary Care and Outcomes Research, Department of Medicine, Stanford University, 615 Crothers Way, Stanford, CA 94305, USA (e-mail: mreitsma@stanford.edu).

Author Contributions MBR, JAS, and JDGF conceptualized and designed the study. MBR analyzed the data. MBR, JAS, and JDGF provided input on data, results, and interpretation. MBR, JAS, and JDGF drafted the manuscript, revised the manuscript for important intellectual content, and approved the final version.

Funding Our global work on household crowding, vulnerability, and transmission of SARS-CoV-2 is supported in part by a gift from the Wadhwani Institute for Artificial Intelligence Foundation. MBR is supported by Stanford's Knight-Hennessy Scholars program. JAS is supported by funding from the Centers for Disease Control and Prevention though the Council of State and Territorial Epidemiologists (NU38OTO00297-02). JAS and JDGF are supported by funding from the National Institute on Drug Abuse (3R37DA01561217S1).

Role of the Funding Source The funders had no role in the study design, management, data analysis, preparation of the manuscript, and decision to submit the manuscript for publication.

Data Availability The datasets generated in this study are available in a GitHub repository: https://github.com/SC-COS$\mathrm{MO} /$ household_exposure_risk.

\section{Declarations:}

Conflict of Interest: The authors declare that they do not have a conflict of interest. 


\section{REFERENCES}

1. Bibbins-Domingo K. This Time Must Be Different: Disparities During the COVID-19 Pandemic. Ann Intern Med. Published online April 28, 2020. doi:https://doi.org/10.7326/M20-2247

2. Selden TM, Berdahl TA. COVID-19 And Racial/Ethnic Disparities In Health Risk, Employment, And Household Composition. Health Affairs. Published online July 14, 2020:https://doi.org/10.1377/hlthaff.2020. 00897

3. Williams DR, Cooper LA. COVID-19 and Health Equity-A New Kind of "Herd Immunity." JAMA. 2020;323(24):2478-2480. doi:https://doi.org/ 10.1001/jama.2020.8051

4. Ruggles S, Flood S, Goeken R, et al. IPUMS USA: Version 10.0 2014-2018 American Community Survey 5-Year Dataset. IPUMS; 2020. https://doi. org/10.18128/D010.V10.0
5. LMI Institute. SOC Codes for CISA Critical Infrastructure Workers. Accessed June 28, 2020. https://www.lmiontheweb.org/more-than-half-of-u-sworkers-in-critical-occupations-in-the-fight-against-covid-19

6. Yan BW, Sloan FA, Dudley RA. How Influenza Vaccination Rate Variation Could Inform Pandemic-Era Vaccination Efforts. J GEN INTERN MED. 2020;35(11):3401-3403. doi:https://doi.org/10.1007/s11606-02006129-x

Publisher's Note: Springer Nature remains neutral with regard to jurisdictional claims in published maps and institutional affiliations. 